\title{
Multiple variations of the tendons of the anatomical snuffbox
}

San San $\underline{T h w i n^{1}}$, MBBS, MMedSc, Fazlin $\underline{\text { Zaini }}^{1}$, MMed Sc, Myo $\underline{\operatorname{Than}^{1}}$, MBBS, MMedSc

INTRODUCTION Multiple tendons of the abductor pollicis longus (APL) in the anatomical snuffbox of the wrist can lead to the development of de Quervain's syndrome, which is caused by stenosing tenosynovitis. A cadaveric study was performed to establish the variations present in the tendons of the anatomical snuffbox in a Malaysian population, in the hope that this knowledge would aid clinical investigation and surgical treatment of de Quervain's tenosynovitis.

METHODS Routine dissection of ten upper limbs was performed to determine the variations in the tendons of the anatomical snuffbox of the wrist.

RESULTS In all the dissected upper limbs, the APL tendon of the first extensor compartment was found to have several (3-14) tendon slips. The insertion of the APL tendon slips in all upper limbs were at the base of the first metacarpal bone, trapezium and fascia of the opponens pollicis muscle; however, in seven specimens, they were also found to be attached to the fleshy belly of the abductor pollicis brevis muscle. In two specimens, double tendons of the extensor pollicis longus located in the third extensor compartment were inserted into the capsule of the proximal interphalangeal joints before being joined to the extensor expansion. In two other specimens, the first extensor compartment had two osseofibrous tunnels divided by a septum that separated the APL tendon from the extensor pollicis brevis tendon.

CONCLUSION Multiple variations were found in the anatomical snuffbox region of the dissected upper limbs. Knowledge of these variations would be useful in interventional radiology and orthopaedic surgery.

Keywords: abductor pollicis longus, extensor pollicis brevis, extensor pollicis longus, tendon

\section{INTRODUCTION}

The presence of multiple tendons of the abductor pollicis longus (APL) is clinically relevant, as it can lead to the development of de Quervain's syndrome, which is caused by stenosing tenosynovitis. ${ }^{(1,2)}$ Normally, there are six extensor compartments in the dorsum of the hand (Fig. 1) - the first compartment contains the APL and extensor pollicis brevis (EPB); the second compartment, the extensor carpi radialis longus and extensor carpi radialis brevis; the third compartment, the extensor pollicis longus (EPL); the fourth compartment, the extensor digitorum and extensor indicis; the fifth compartment, the extensor digiti minimi; and the sixth compartment, the extensor carpi ulnaris.

The anatomical snuffbox of the wrist is bound by the first and third extensor compartments. It contains the radial artery, and its roof is crossed by the cephalic vein. The anatomical snuffbox is significant, as the APL tendon (tendon of the first compartment) can get inflamed, usually due to overuse, particularly during gripping/grasping. In such cases, patients usually complain of pain at the dorsolateral aspect of the wrist, radiating toward the thumb or lateral forearm, or both. ${ }^{(2)}$ While variations in the anatomical snuffbox of the wrist may cause clinically significant problems, such variations may also serve as a potential source of transplant material, especially for tendon reconstructions. ${ }^{(3)}$ Thus, we studied variations of the tendons of the anatomical snuffbox in the hope that more precise knowledge of these tendons would aid clinical investigation and effective surgical treatment of de Quervain's tenosynovitis.

\section{METHODS}

This study was carried out at the Department of Anatomy, Faculty of Medicine, Universiti Kuala Lumpur-Royal College of Medicine Perak, Malaysia. Routine dissection of ten upper limbs was performed according to the procedure described in Cunningham's Manual of Practical Anatomy.(4) The posterior compartment of the forearm and the dorsum of the hand, together with the extensor compartments, were dissected and studied for variations of the tendons of the APL, EPB and EPL. Photographic documentation was done using a Sony DSC-T30 camera (Sony [Malaysia] Sdn Bhd, Kuala Lumpur, Malaysia).

\section{RESULTS}

The APL tendon of the first extensor compartment was observed to have several (varying from 3 to 14) tendon slips in all the upper limbs (Fig. 2). The APL tendon slips in all the upper limbs were inserted into the base of the first metacarpal bone, trapezium and fascia of the opponens pollicis muscle. In seven

${ }^{1}$ Anatomy Department, Universiti Kuala Lumpur - Royal College of Medicine Perak, Ipoh, Malaysia

Correspondence: Dr San San Thwin, Associate Professor, Anatomy Department, Universiti Kuala Lumpur - Royal College of Medicine Perak, 3 Jalan Greentown, 30450 Ipoh, Perak, Malaysia. santhwin@yahoo.com 


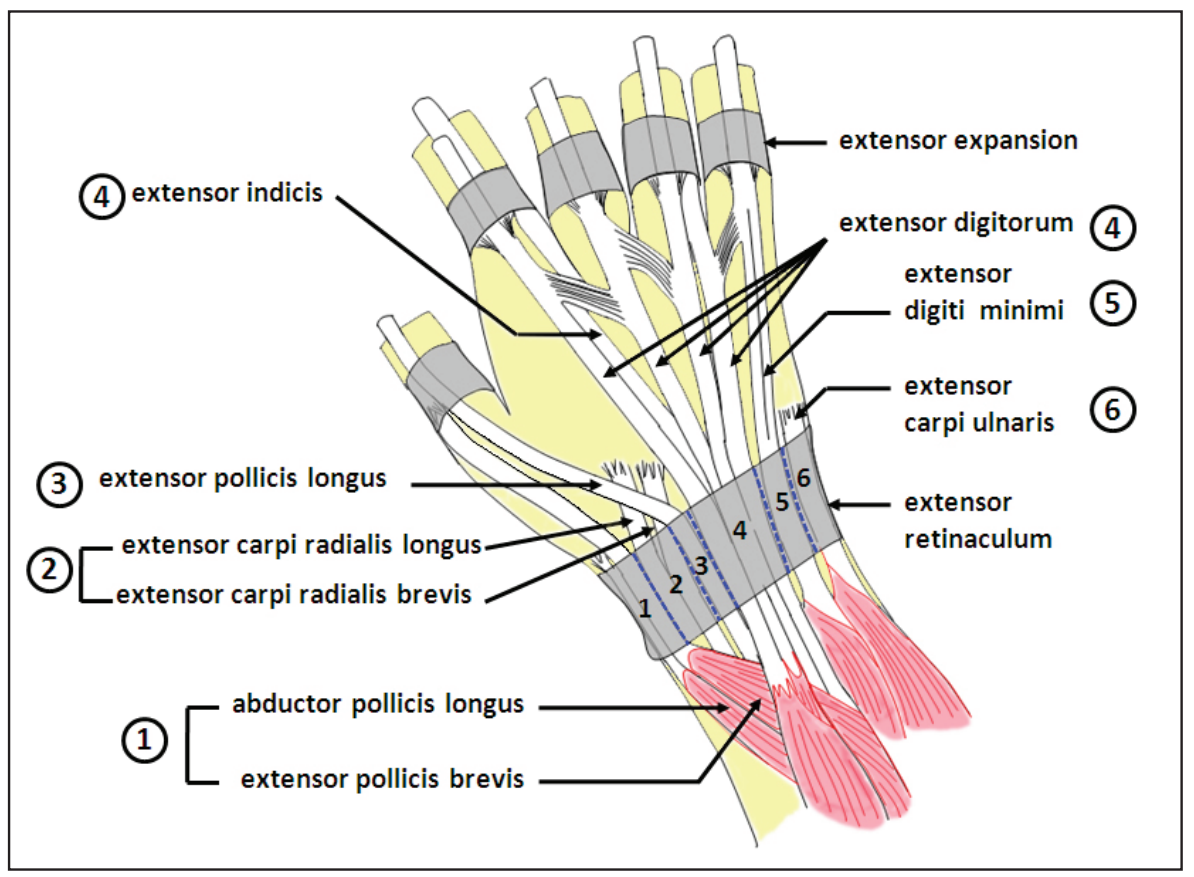

Fig. 1 Schematic diagram shows the extensor compartments (1-6) of the dorsum of the hand and their contents.

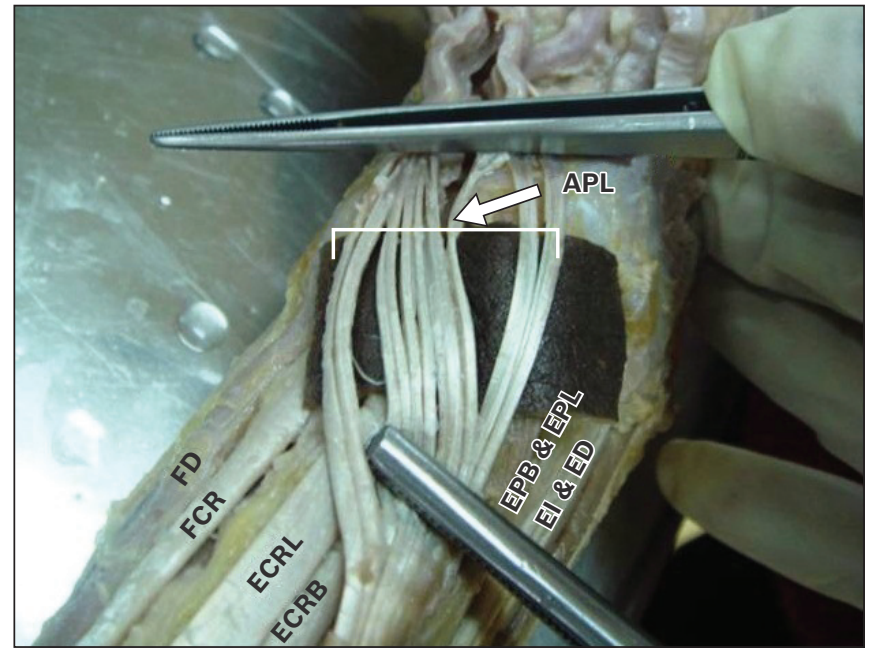

Fig. 2 Photograph of the left upper limb of specimen 10 shows the APL with 14 tendon slips. Some tendons of the extensor and flexor compartments are also seen.

APL: abductor pollicis longus; ECRB: extensor carpi radialis brevis; ECRL: extensor carpi radialis Iongus; ED: extensor digitorum; EI: extensor indicis; EPB: extensor pollicis brevis; EPL: extensor pollicis longus; FCR: flexor carpi radialis; FD: flexor digitorum

specimens (specimens 1-4 and 7-9), the APL tendon slips were also found to be inserted into the fleshy belly of the abductor pollicis brevis (APB) muscle (Fig. 3).

In specimens 9 and 10 , the tendon of the EPL, which is usually single, was found to be double and located in the third extensor compartment. The tendons were inserted into the capsule of the proximal interphalangeal joints before being joined to the extensor expansion (Fig. 4). No variation was found in the EPB muscle. In specimens 4 and 5, the first extensor compartment was found to have two osseofibrous tunnels divided by a septum that separated the tendon of the APL from that of the EPB (Fig. 5).

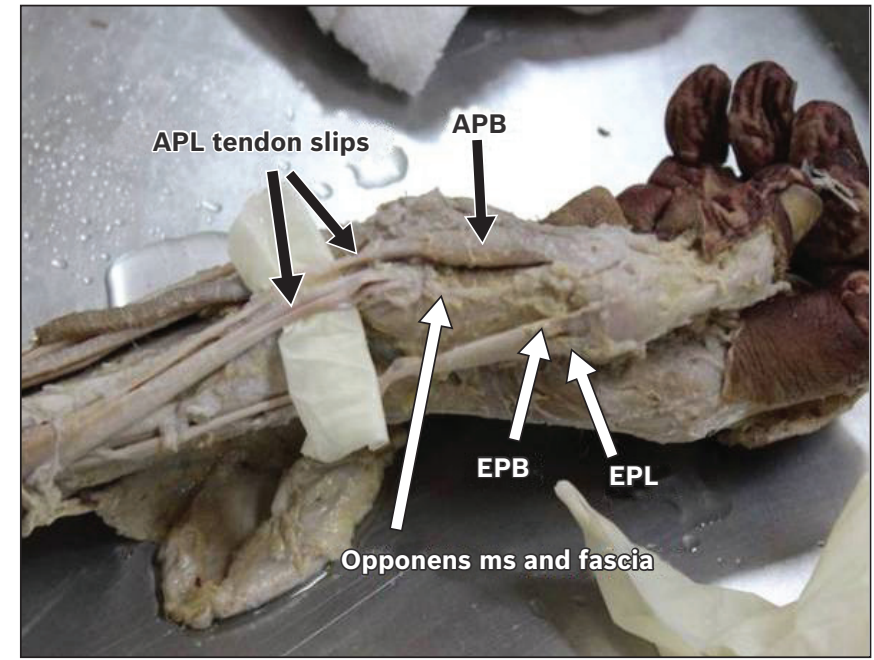

Fig. 3 Photograph of the right upper limb of specimen 1 shows the insertion of the APL tendon into the fleshy belly of the APB. Other tendinous slips of the APL show attachment to the fascia of the opponens pollicis muscle.

APL: abductor pollicis longus; APB: abductor pollicis brevis; EPB: extensor pollicis brevis; EPL: extensor pollicis longus

\section{DISCUSSION}

In this study that focused on multiple variations of the anatomical snuffbox of the wrist, we found supernumerary tendon slips of the APL, ranging from 3 to 14 slips, in the ten upper limb specimens dissected. Multiple variations of the APL are common; ${ }^{(1)}$ five, $^{(3)}$ six $^{(5)}$ and nine ${ }^{(1)}$ APL tendon slips have previously been reported. Mansur et al have suggested that these supernumerary tendons are clinically important in reconstructive surgery. ${ }^{(1)}$ Mehta et al proposed that when supernumerary tendons were present, injury to a single tendon would not disturb the normal function of the thumb, as the additional tendons would supplement the functional capacity 


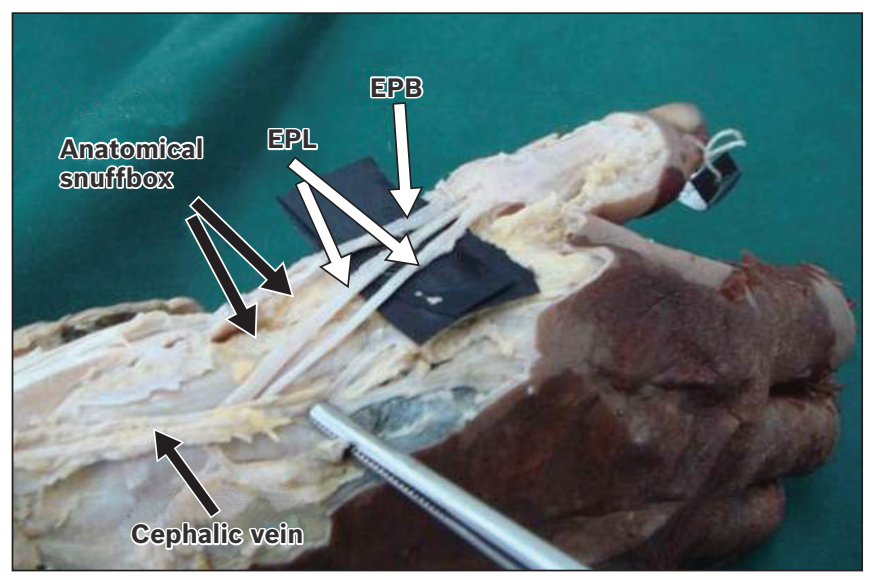

Fig. 4 Photograph of the right upper limb of specimen 10 shows the EPL with two tendons. The anatomical snuffbox, together with the cephalic vein, is also shown.

EPB: extensor pollicis brevis; EPL: extensor pollicis longus

of APL tendons. ${ }^{(6)}$ According to Mansur et al, the presence of multiple APL tendons may have functional significance in the development of de Quervain's stenosing tenosynovitis. ${ }^{(1)}$ They also suggested that multiple tendons of the APL might modify the force component, thus altering the mechanics of the thumb. ${ }^{(1)}$

In this current study, the insertion of the APL tendon slips in all the upper limbs was at the base of the first metacarpal bone, trapezium and fascia of the opponens pollicis muscle. Apart from the aforementioned insertions, the tendon slips in seven specimens were found to be also attached to the fleshy belly of the APB. Similar findings have been reported by Kocabiyik et al. ${ }^{(7)}$ Likewise, Mansur et al had also reported APL tendon insertions at the lateral and anterolateral sides of the base of the first metacarpal bone, trapezium, opponens pollicis, APB and thenar fascia. ${ }^{(1)}$

During the early developmental period, the APL tendon is divided into three strips, with the middle strip inserted into the trapezium and the dorsal strip attached to the first metacarpal bone. The palmar strip, which is connected to the opponens pollicis, is disconnected when new connections with the APB are established. ${ }^{(8)}$ It is possible that such persistent tendinous patterns can lead to multiple tendons being observed later in life. ${ }^{(6)}$

We found double tendons of the EPL in the third extensor compartment of two specimens. Nishijo et al had also reported duplicated EPL tendons that passed medial to the APL and were inserted into the interphalangeal joint. ${ }^{(9)}$ Although a normal tendon is usually strong, the EPL may rupture due to added friction imposed on it, for example in Colles fracture, ${ }^{(10)}$ which is normally treated by tendon transfer surgery. ${ }^{(11)}$ However, it is possible that in individuals who have an additional tendon, a ruptured tendon may actually be compensated by the second tendon, thus maintaining proper functioning of the thumb.

The first extensor compartment usually contains the tendons of the EPB and APL in a single compartment. In two

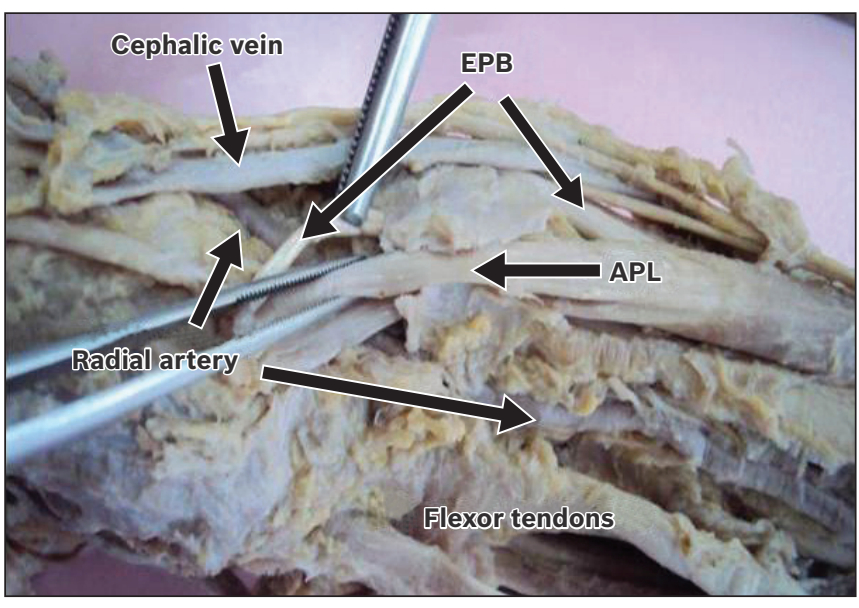

Fig. 5 Photograph of the left upper limb of specimen 5 shows the tendons of the APL and EPB within separate tunnels in the first extensor compartment. Also shown are the radial artery and cephalic vein in the anatomical snuffbox.

APL: abductor pollicis longus; EPB: extensor pollicis brevis

of the specimens in our study, the first extensor compartment was divided by a septum into two separate tunnels instead of a single compartment. Similarly, a study by Nayak et al found that in $34.6 \%$ of the upper limbs $(n=54)$, the tendons of the EPB were separated from the APL tendons by an osseofibrous septum in the first extensor compartment. ${ }^{(12)}$ Kulthanan and Chareonwat have suggested that the presence of multiple compartments in the first extensor compartment may be clinically significant, as it may predispose one to de Quervain's syndrome. (2) The authors reported that the presence of subcompartments was more commonly seen in patients with de Quervain's syndrome than in the normal population $(58 \%$ vs. $37 \%)^{(2)}$ Septation in the first extensor compartment may also affect nonoperative treatment of the wrist, as the septum may limit the delivery of any injected steroid into these compartments. ${ }^{(13)}$

To conclude, multiple variations, including supernumerary tendon slips of the APL, insertion of the APL to the APB, double EPL tendons and the presence of fibrous septum in the first extensor compartment, were seen in the anatomical snuffbox region of the upper limbs dissected in our study. The existence of multiple tendons and the presence of septation in the compartment might be clinically significant for the treatment of de Quervain's syndrome. Detailed knowledge of these variations will not only aid interventional radiology (e.g. help radiologists in diagnosing tendon rupture), but also orthopaedic surgery (e.g. during tendon reconstructive surgery).

\section{ACKNOWLEDGEMENTS}

The authors thank Prof Osman Ali, A/Prof Cheah Tong Soon, A/Prof Noorzaid Muhamad, Dr Sandheep Sugathan and Puan Resni Mona Abdullah for their guidance and help. We also acknowledge the assistance rendered by Puan Nikki Amura Zanial Abidin, Puan Zuraida Salleh, Cik Noor Huda Aminallah and Cik Jamaatul Akma Abu Ngamar during the preparation of this article. 


\section{REFERENCES}

1. Mansur DI, Krishnamurthy A, Nayak SR, et al. Multiple tendons of abductor pollicis longus extensors. Int J Anat Var 2010; 3:25-6.

2. Kulthanan $\mathrm{T}$, Chareonwat B. Variations in abductor pollicis longus and extensor pollicis brevis tendons in the Quervain syndrome: a surgical and anatomical study. Scand J Plast Reconstr Surg Hand Surg 2007; 41:36-8.

3. Akan M, Gideroglu K, Cakir B. Multiple tendons of the abductor pollicis longus muscle. Hand Surg 2002; 7:289-91.

4. Cunningham DJ, Romanes GJ. Cunningham's Manual of Practical Anatomy. 15th ed. New York: Oxford University Press, 1986.

5. Nayak SR, Krishnamurthy A, Pai MM, et al. Multiple variations of the extensor tendons of the forearm. Rom J of Morpho and Embryol 2008; 49:97-100.

6. Mehta V, Arora J, Suri RK, Rath G. A rare quadruplicate arrangement of abductor pollicis longus tendons: anatomical and clinical relevance. Clinics (Sao Paulo) 2009; 64:153-5.

7. Kocabiyik N, Tatar I, Yalcin B, Yazar F, Ozan H. Tendon variations of extensor digitorum and abductor pollicis longus muscles. Int J Anat Var 2009; 2:54-6.

8. Cihák R. Connections of the abductor pollicis longus and brevis in the ontogenesis of the human hand. Folia Morphol (Praha) 1972; 20:102-5.

9. Nishijo K, Kotani H, Miki T, Senzoku F, Ueo T. Unusual course of the extensor pollicis longus tendon associated with tenosynovitis, presenting as de Quervain Disease--a case report. Acta Orthop Scand 2000; 71: 426-28.

10. Trevor D. Rupture of the extensor pollicis longus tendon after Colles fracture. J Bone Joint Surg Br 1950; 32-B:370-5.

11. Magnussen PA, Harvey FJ, Tonkin MA. Extensor indicis proprius transfer for rupture of the extensor pollicis longus tendon. J Bone Joint Surg $\mathrm{Br}$ 1990; 72:881-3.

12. Nayak SR, Hussein M, Krishnamurthy A, et al. Variation and clinical significance of extensor pollicis brevis: a study in South Indian cadavers. Chang Gung Med J 2009; 32:600-4.

13. Witt J, Pess G, Gelberman RH. Treatment of de Quervain tenosynovitis: a prospective study of the results of injection of steroids and immobilization in a splint. J Bone Joint Surg Am 1991; 73:219-22. 